\title{
Ambulatory Function in Men with and without HIV Infection: Association with Cardiorespiratory Fitness
}

Jeffrey Beans ${ }^{1,2}$, Troy Stevenson ${ }^{1,2}$, Leslie I Katzel ${ }^{1,2}$, John D Sorkin ${ }^{1,2}$, Alberta L Warner ${ }^{3}$, Stephen S. Gottlieb ${ }^{1,2}$, Kristina Crothers ${ }^{4}$, Adeel A Butt $^{5,6}$, David A Leaf ${ }^{3}$, Matthew S Freiberg ${ }^{5}$ and Krisann K Oursler ${ }^{1,2 *}$

${ }^{1}$ University of Maryland School of Medicine, USA

${ }^{2}$ Baltimore Veterans Affairs Medical Center and Geriatrics Research Education and Clinical Center, USA

${ }^{3}$ U.C.L.A. Schools of Medicine and Greater Los Angeles V.A. Healthcare System, USA

${ }^{4}$ University of Washington, Seattle, Washington, USA

${ }^{5}$ University of Pittsburgh School of Medicine, USA

${ }^{6}$ Sheikh Khalifa Medical City, Abu Dhabi

\begin{abstract}
Objective: The study purpose was to compare ambulatory function in men with and without HIV infection, and test the association with aerobic exercise capacity.

Methods: We conducted a cross-sectional study of 45 HIV-infected men and 37 age and race-matched HIVuninfected men at the Baltimore VA Medical Center. Participants performed cardiorespiratory exercise testing, sixminute walk (6-MW) and the 400-meter long distance corridor walk (LDCW) as part of a study of veterans without history of cardiovascular disease.

Results: The mean (SD) age was 55 (6) years. Among the 82 male participants, 98\% were African American race. The 6-MW distance correlated with aerobic exercise capacity (VO2peak) in both HIV-infected subjects ( $r=0.50$ $p<.001)$ and uninfected subjects $(r=0.57, p<0.001)$. Duration in the 400 meter LDCW inversely correlated with VO2peak in HIV-infected $(r=-0.52, p<.001)$ and uninfected subjects $(r=-0.48, p<.001)$. In linear regression models the variance in VO2peak explained by the endurance walk test was similar for the 6-MW and LDCW across HIV groups (all $p>0.1)$. Between HIV groups, there was a significant difference in LDCW $(p=0.01)$ but not in 6-MW $(p=0.3)$. Conclusions: In HIV-infected men without known CVD the 6-MW and LDCW provide similar estimates of aerobic exercise capacity. The findings are comparable to uninfected men with similar demographic and clinical characteristics, and support endurance walk tests to estimate aerobic exercise capacity in HIV-infected patients.
\end{abstract}

Keywords: HIV; Ambulatory Function; Cardiorespiratory fitness

\section{Introduction}

Ambulatory function predicts mortality and mobility disability in older adults [1-6]. However, little information is available in HIVinfected adults despite that fact that an estimated half of the HIVinfected individuals living in the United States will be $50+$ years of age in the next five years [7]. Ambulatory function measured by selfpaced walking tests assesses both mobility and capacity to perform real-life daily activities in adults with a wide range of limitations. Longer distance tests, such as the six-minute walk (6-MW) and the long-distance corridor walk (LDCW), correlate with aerobic exercise capacity (VO2peak) measured by cardiorespiratory testing in patients with congestive heart failure [5] and chronic obstructive lung disease (COPD) [8]. These tests are therefore referred to as endurance walk tests to differentiate them from short-distance timed walks (e.g., 4-meter) that focus on mobility limitations and gait speed. Short-distance timed walks are also associated with leg strength [9], but are more likely to be limited by a ceiling effect in individuals with high level of physical function.

Richert et al. recently reported poor performance in the six-minute walk (6-MW) in a quarter of 324 HIV-infected adults (median age 48 years) based on normative data, generated by Enright's reference equation using age, sex, weight, and height [10]. Esposito and colleagues found the 6-MW distance to be 5\% lower than expected in 27 younger men [11] using the same reference equation [12]. We reported a $8 \%$ lower 6-MW distance in 32 older HIV-infected men and a moderate correlation between the 6-MW distance and VO2peak [13]. Erlandson et al. recently showed a $62 \%$ agreement between the LDCW and short physical performance battery (SPPB) in assessment of physical function, but did not include exercise testing [14]. These studies are limited by lack of a HIV uninfected comparison group.

Compared to endurance walking tests, cardiorespiratory testing offers the advantage of precise physiologic data with information on degree of effort and workload. Oxygen utilization at peak exercise (VO2peak) is a gold standard for aerobic exercise capacity and is significantly impaired in HIV-infected adults [15], which is associated with increased age [13], hypertension [16], hyperlactatemia [17], and low CD4 cell count [18]. However, cardiorespiratory testing requires expensive equipment and specially trained staff.

In contrast to the endurance walk tests, performance in shortdistance timed walks in HIV-infected adults on successful antiretroviral therapy (ART) is not different from uninfected adults. The Women's Interagency HIV Study (WIHS) reported that the median time to complete the 4-meter walk test was similar in HIV-negative women

*Corresponding author: Dr. Krisann K. Oursler, Baltimore Veterans Affairs Medical Center and Geriatrics Research Education and Clinical Center, $10 \mathrm{~N}$ Greene Street, BT/18/GR, Baltimore, MD 21201, USA, Tel: 410 605-7185; Fax: 410-605-7709; E-mail: Krisann.oursler@va.gov

Received March 29, 2013; Accepted May 10, 2013; Published May 18, 2013

Citation: Beans J, Stevenson T, Katzel LI, Sorkin JD, Warner AL, et al. (2013) Ambulatory Function in Men with and without HIV Infection: Association with Cardiorespiratory Fitness. J AIDS Clinic Res S9: 003. doi:10.4172/2155-6113.S9-003

Copyright: @ 2013 Beans J, et al. This is an open-access article distributed under the terms of the Creative Commons Attribution License, which permits unrestricted use, distribution, and reproduction in any medium, provided the original author and source are credited. 
(4.24 secs) and HIV-infected women with CD4+ counts $\geq 500$ cells/ $\mathrm{mm}^{3}$ (4.28 secs) [19]. The corresponding gate speed, greater than one meter/sec, in these middle-aged women (median age 38 years (HIV -) and 4lyears $(\mathrm{HIV}+$ ) is higher than threshold values for frailty in elderly women [20] and cut-off values that predict mortality [21] and mobility disability [1] in cohorts of uninfected elderly adults. However, WIHS HIV-infected women with CD4 cell count $<100$ cells $/ \mathrm{mm}^{3}$ did meet these criteria with a significantly longer 4 -meter walk test $(5.08$ secs) [19].

Ambulatory function is a feasible and validated geriatric construct, which can be used to investigate risk for physical disability, and all cause mortality in older HIV-infected adults. Current evidence in HIV-infected adults on successful ART suggests a deficit in walking endurance rather than mobility. Our study objective was to perform endurance walk tests in men with and without HIV infection, and test the association with aerobic exercise capacity.

\section{Methods}

\section{Subjects}

Men enrolled in the Veterans Aging Cohort Study (VACS) at the Baltimore VA Medical Center were recruited for the Cardiovascular Sub-study (CVD-VACS), which included cardiorespiratory testing and assessment of physical function. VACS is a multicenter prospective longitudinal observational study of HIV-infected veterans from HIV Clinics with age, race, and site group matched HIV uninfected veterans from General Medicine Clinics [22]. The CVD-VACS study excluded VACS participants with known cardiovascular disease (CVD), specifically: 1) history of prevalent coronary artery disease (myocardial infarction, angioplasty, stent placement, coronary artery bypass surgery or angina symptoms requiring medication);2) history of peripheral vascular disease with claudication; 3 ) history of cerebral vascular disease (stroke or transient ischemic attack); and 4) stage 3 or 4 congestive heart failure. Participants with severe musculoskeletal disease, which required assist device for ambulation, were also excluded for safety reasons. Among the first 84 Baltimore site CVD-VACS participants, an endurance walking test was not completed in two subjects, with a resulting analytic set of 82 participants (45 HIV-infected, 37 uninfected participants). Participants provided written informed consent for both VACS and CVD-VACS, which were approved by the University of Maryland Baltimore Institutional Review Board and Baltimore VA Research and Development Committees.

\section{Ambulatory function}

The six-minute walk (6-MW) and long distance corridor walk (LDCW) tests were performed in random order on the same day in a corridor with pre-measured courses marked with cones. Sitting heart rate and blood pressure were measured immediately before and after each test. The second test followed the first after the participant's heart rate returned to its resting rate. Pulse oximetry and heart rate were not monitored while the participant walked. For the six-minute walk (6MW) participants were instructed to cover as much distance as possible in six minutes by walking laps over a 100 -feet course, based on the American Thoracic Society protocol [23]. Encouragement was limited to time splits: the time remaining after each minute and when subjects had 30 and 10 seconds remaining. Distance was measured to the nearest foot and then converted to meters. For the long distance corridor walk (LDCW) test subjects first completed a 2-minute warm up prior to the 400-meter timed walk [24]. The subjects were instructed to walk 10 laps around a 40 meter course marked by cones as quickly as possible without running. Encouragement was limited to laps completed and laps remaining. Time to walk the 400 meters was measured in minutes and seconds with a stopwatch.

\section{Cardiorespiratory exercise testing}

Subjects performed cardiorespiratory exercise testing (CPET) on a motorized treadmill using the modified Bruce protocol with open circuit spirometry to measure gas exchange (oxygen and carbon dioxide) [15]. The modified Bruce protocol starts at a speed of $1.7 \mathrm{mph}$ and $0 \%$ incline with workload increasing at standardized three minute intervals, incline only initially, then by both incline and speed. The test was terminated according to American College of Sports Medicine (ACSM) safety criteria [15] or when subject reported volitional exhaustion. Oxygen utilization, carbon dioxide production, and minute ventilation values were collected breath-by-breath with a Quark CPET and averaged at 10-second intervals. Peak oxygen utilization (VO2peak) was calculated as the average of the highest three 10-second interval measurements near test termination.

\section{Clinical and survey data}

The VACS coordinating center provided clinical information on past medical history based on the electronic medical record as previously described [22], which was confirmed by history and physical exam on enrollment into CVD VACS. Data on cigarette smoking and illicit drug use was obtained from the annual VACS participant survey. During the research visit, current medication list was reviewed, height and weight were measured and used to calculate body mass index (BMI). Estimated 6-MW distance was calculated based on the formula for men by Enright (1140- 5.61(BMI) -6.94(age)) [12].

\section{Statistical analyses}

Differences in subject characteristics and physical function in the HIV-infected and uninfected groups were determined by Student's t-test or chi-squared test. Pearson's correlation and linear regression models tested the association between VO2peak and performance in the 6-MW and LDCW. Separate analyses were performed for the HIVinfected and uninfected subjects. The square root of the mean square error (RMSE), a measure of variance in the dependent variable adjusted for the independent variable, was calculated for each bivariate model. Differences in slopes (beta coefficients) and RMSE between models were tested using the F-statistic. Analyses were performed with STATA statistical software (Stata 9.0; STATACorp, College Station, TX).

\section{Results}

The majority of HIV-infected subjects were receiving combination antiretroviral therapy with a non-detectable HIV-1 viral load $(<400$ copies/ml) in $91 \%$ and a high CD4 cell count (median (IQR):445 (265531). There was no difference in age between the HIV-infected and uninfected men (mean \pm SE, years: $54.4 \pm 6.3$ vs. $54.7 \pm 6.2, \mathrm{p}=0.8$ ) or race (Table 1). The prevalence of common comorbid conditions was comparable between groups. Sixty-one percent of the population had a history of hepatitis C infection, 71\% had hypertension (Table 1). Between groups the use of antihypertensive medication that could affect heart rate results was similar (beta-blockers 10\%; non-dihydropyridine calcium channel blockers $11 \%$; $>0.6$ ). There was a non-significant trend for the uninfected men to be more likely to be obese or overweight (Body Mass Index $(\mathrm{BMI})>25.0 \mathrm{~kg} / \mathrm{m}^{2}$ ) than the HIV-infected men.

Overall, there was a strong correlation between the 6-MW and LDCW ( $r=-0.85, \mathrm{p}<.001)$, with similar results in the HIV-infected and uninfected men. There was no difference in performance in the 6-MW 
Citation: Beans J, Stevenson T, Katzel LI, Sorkin JD, Warner AL, et al. (2013) Ambulatory Function in Men with and without HIV Infection: Association with Cardiorespiratory Fitness. J AIDS Clinic Res S9: 003. doi:10.4172/2155-6113.S9-003

Page 3 of 5

between HIV-infected and uninfected participants (Table 2). Using Enright's reference equation, the expected 6-MW (mean meters $( \pm \mathrm{SD})$ was $617( \pm 48)$ and $598( \pm 47)$ in HIV infected and uninfected groups, respectively. Time for the 400-meter LDCW in the HIV-infected subjects was a mean 18 seconds shorter than the uninfected subjects.

VO2peak had a moderate and significant correlation with the 6-MW distance (meters) and 400-meter walk LDCW duration (minutes) in both subject groups (Figure 1). The 6-MW explained 25\% and $32 \%$ of the variance in VO2 peak in HIV-infected and uninfected subjects, respectively (Table 3 ). The 400 -meter walk explained $27 \%$ and $23 \%$ of the variance in VO2 peak in HIV-infected and uninfected subjects, respectively. Comparison between the HIV-infected and uninfected models showed no significant difference in $\triangle \mathrm{VO} 2$ peak by unit change in ambulatory performance for either the 6-MW $(p=0.9)$ or the LDCW ( $\mathrm{p}=0.8$ ) (Table 3). There was no significant difference in the corresponding values of the SEE across HIV groups ( $p>0.1)$. Results were unchanged in linear regression models adjusted for BMI (data not shown).

\section{Discussion}

This study presents novel results on ambulatory function in patients with and without HIV infection who have similar demographic and clinical characteristics. Among these men without history of CVD, ambulatory function was not significantly different by HIV status. The relationship between endurance walk tests and VO2peak supports use of either the 6-MW or LDCW in HIV research to estimate aerobic exercise capacity. Endurance walk tests are valuable prognostic tools in HIV uninfected geriatric patient populations. Further investigation in larger and more diverse group of HIV-infected patients is needed to determine their clinical value.

This study offers the first direct comparison of endurance walk tests between HIV-infected and uninfected adults and includes validation with cardiorespiratory exercise testing. Despite the small and homogeneous study population, the findings have several important

\begin{tabular}{|c|c|c|c|}
\hline Characteristics & $\begin{array}{l}\text { HIV-infected } \\
\begin{array}{c}\mathrm{N}=45 \\
\mathrm{n}(\%)\end{array}\end{array}$ & $\begin{array}{l}\text { HIV-uninfected } \\
\qquad \begin{array}{c}\mathrm{N}=37 \\
\mathrm{n}(\%)\end{array}\end{array}$ & p-value* \\
\hline \multicolumn{4}{|l|}{ Age, years: } \\
\hline$<50$ & $6(13.3)$ & $8(21.6)$ & 0.3 \\
\hline $50-60$ & $30(66.7)$ & $22(59.5)$ & \\
\hline$>60$ & $9(20.0)$ & 7 (18.9) & \\
\hline Race, African American & $45(100)$ & $36(97.3)$ & 0.2 \\
\hline \multicolumn{4}{|l|}{ Body Mass Index (BMI): } \\
\hline$<25 \mathrm{~kg} / \mathrm{m}^{2}$ & $23(51.1)$ & $12(32.4)$ & 0.09 \\
\hline$\geq 25 \mathrm{~kg} / \mathrm{m}^{2}$ & $22(48.9)$ & $25(67.6)$ & \\
\hline Smoker, current & $31(69.0)$ & $21(56.8)$ & 0.3 \\
\hline \multicolumn{4}{|l|}{ Comorbidity: } \\
\hline Diabetes & $12(26.7)$ & $7(18.9)$ & 0.4 \\
\hline Hepatitis C Infection & $32(71.1)$ & $18(55.6)$ & 0.1 \\
\hline Hypertension & $31(68.9)$ & $27(73.0)$ & 0.7 \\
\hline $\begin{array}{l}\text { Chronic Pulmonary } \\
\text { Disease }\end{array}$ & $9(20.0)$ & $11(29.7)$ & 0.3 \\
\hline Dyslipidemia & $16(36.4)$ & $8(25.8)$ & 0.3 \\
\hline Anemia, $(\mathrm{Hgb}<13 \mathrm{~g} / \mathrm{dl})$ & $11(24.4)$ & $14(37.8)$ & 0.2 \\
\hline
\end{tabular}

Table 1: Characteristics of HIV-infected and uninfected Men

\begin{tabular}{lccc}
\hline Results & HIV-infected & HIV-uninfected & p-value \\
\hline 6-MW (Mean (SE)): & $\mathrm{N}=45$ & $\mathrm{~N}=37$ & \\
Distance, meters & $545(10)$ & $531(11)$ & 0.3 \\
Heart rate maximum, beats/min & $113(3)$ & $115(3)$ & 0.7 \\
400-meter LDCW (Mean (SE)): & $\mathrm{N}=43$ & $\mathrm{~N}=35$ & \\
Time, seconds & $258.5(4.9)$ & $276.3(5.5)$ & 0.02 \\
Heart rate maximum, beats/min & $115(3)$ & $116(3)$ & 0.9
\end{tabular}

Six-minute walk (6-MW); long distance corridor walk (LDCW)

* Student's t-test

Table 2: Ambulatory function in HIV-infected and uninfected men.

implications. First, the results highlight the importance of a HIV uninfected comparison group when investigating non-HIV related outcomes. Several recent functional performance studies, showed reduced 6-MW distance $[10,13,19]$ or LCDW time [14] in HIV-infected adults using normative data. In this study we found no significant difference in the 6-MW distance between HIV-infected on successful ART and uninfected men with similar age-related comorbidity, even with adjustment for BMI. This is not to say that AIDS does not adversely and significantly affect ambulatory function via mechanisms of muscle mass loss [25]. The prior reports of poor functional performance in HIV-infected adults with high CD4 cell count based on normative data is likely affected by differences in socioeconomic and clinical characteristics between the HIV-infected patients and the reference group. This possibility is supported by our finding that the estimated 6-MW distance in the HIV-infected adults was $12 \%$ lower than expected, based on a standard reference equation This mean difference of 73 meters represents both a statistical and clinical meaningful lower 6-MW distance. In contrast, the direct comparison of 6-MW distance between HIV groups found a mean difference of 14 meters, which is neither statistically or clinically significant.

Our findings in this select group of men raise the question of the effect of age-related comorbidity on function in HIV-infected adults. Although we found no difference in ambulatory function between HIV groups, we excluded patients with known CVD and severe congestive heart failure as part of the CVD VACS study. Therefore our findings cannot be generalized to HIV-infected men at large; HIV-infected adults may still have a greater risk for poor ambulatory function due to increased disease burden of age-related conditions that are more common in HIV-infected adults. Further, disease severity may affect function. We previously reported that HIV-infected and uninfected patients in the Veterans Aging Cohort Study (VACS) had similar degree of limitations with physical activities [26]. However, when stratified by chronic lung disease, the HIV-infected patients had significantly lower reported physical function, with adjustment for demographics and smoking [27]. Future research with measures of ambulatory function in large diverse cohorts of HIV infected and uninfected older adults is needed to address these questions and provide insight into the clinical factors contributing to poor physical function in adults with chronic HIV infection.

To our knowledge, this is the first study to measure the LDCW in both HIV-infected and uninfected adults, and compare results with the 6-MW test. We found the time to complete the 400 -meter walk was shorter in the HIV-infected men (4.31 minutes) compared to the uninfected men (4.63 minutes). Although this mean difference was statistically significant, it may not be clinically significant [28]. In healthy elderly men enrolled in the Baltimore Longitudinal Study of 

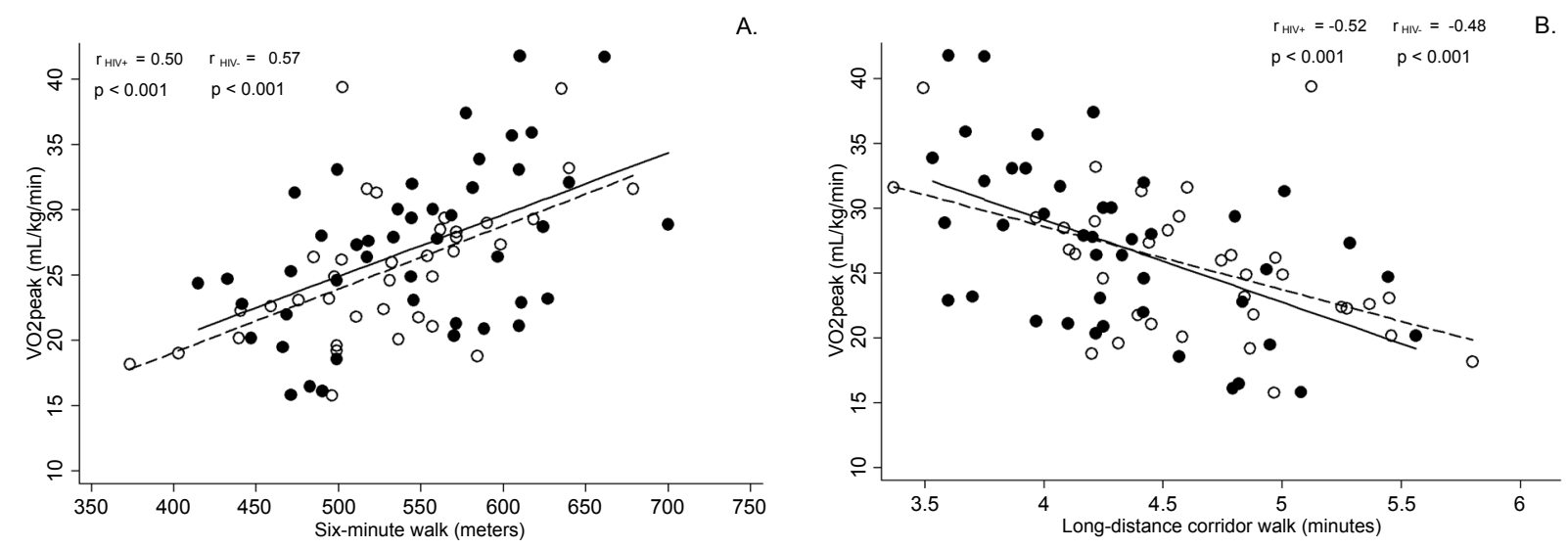

HIV-infected (HIV +, solid circle and line) versus uninfected (HIV -, circle and dotted line) men Panel A. Six-minute walk

Panel B. 400-meter long-distance corridor walk.

Figure 1: Association of self-paced walking tests with VO2peak ( $\mathrm{ml} / \mathrm{kg} / \mathrm{min})$.

\begin{tabular}{|c|c|c|c|c|c|c|c|c|c|c|c|}
\hline \multirow[b]{3}{*}{ Subjects } & \multicolumn{6}{|c|}{ Six-minute walk, meters } & \multicolumn{5}{|c|}{ 400-meter LDCW, minutes } \\
\hline & \multirow[b]{2}{*}{$\mathrm{N}$} & \multicolumn{2}{|c|}{ Model } & \multicolumn{2}{|c|}{ Slope (ml/kg/min/m) } & \multirow[b]{2}{*}{$p^{*}$} & \multicolumn{3}{|c|}{ Model } & \multicolumn{2}{|c|}{ Slope $(\mathrm{ml} / \mathrm{kg} / \mathrm{min} / \mathrm{min})$} \\
\hline & & $\mathrm{R}^{2}$ & RMSE & $\beta$ & SE & & $\mathrm{N}$ & $\mathrm{R}^{2}$ & RMSE & $\beta$ & SE \\
\hline HIV-infected & 45 & 0.25 & 5.51 & $0.047^{\dagger}$ & 0.013 & 0.9 & 43 & 0.27 & 5.56 & $-6.35^{\dagger}$ & 1.64 \\
\hline Uninfected & 37 & 0.32 & 4.56 & $0.048^{+}$ & 0.012 & & 35 & 0.23 & 4.90 & $-4.87^{\dagger}$ & 1.54 \\
\hline
\end{tabular}

* difference in slope between models by the F-statistic

${ }^{\dagger} p$-value $<0.01$ for linear regression $\beta$ coefficient

RMSE, root mean squared error

Table 3: Association of VO2peak ( $\mathrm{ml} / \mathrm{kg} / \mathrm{min}$ ) with performance in the six-minute walk and the 400-meter long-distance corridor walk (LDCW) by linear regression in HIVinfected and uninfected men.

Aging the mean time in the 400 -meter walk is 4.07 minutes [24]. In community dwelling elderly adults each increased minute to perform the 400 -meter walk is associated with a $29 \%$ increased mortality risk and 52\% increased risk of mobility limitations [3]. Erlandson and colleagues recently performed the LDCW in a clinic-based cohort of 359 HIV-infected patients aged 45-65 years who received ART for at least 6 months and were $85 \%$ male [29]. Patients were not excluded based on comorbid conditions, with $18 \%$ (N 66) of the participants reporting recurrent falls in the prior year. Performance in the 400-meter walk among HIV-infected patients without fall history is comparable to our findings (4.38 minutes). However, the 400 -meter time in those with recurrent falls is significantly slower (5.0 minutes), despite a comparable high mean CD4 cell count $>500$. Multiple comorbidities were predictive of falling history.

Performance in endurance walk tests is used as a surrogate for aerobic exercise capacity in patients at high risk for physical disability. Ross and colleagues conducted a meta-analysis which included over a thousand patients with diverse cardiorespiratory conditions from 11 different studies and found that the association between 6-MW and VO2peak was consistent and significant, regardless of differences in the 6-MW and exercise protocols [30]. Among all the studies, the average standard error of the estimate (3.82) was $27 \%$ of the mean VO2peak. Their results demonstrated that the greatest source of error was random within subject error, and not variability in testing methodology. Our results corroborate these findings for the first time in a study of HIVinfected patients that includes a control patient group from the same site and using same testing protocols. The average standard error of the estimate (RMSE) of our 6-MW and VO2 models was not significantly different between HIV groups and represented $18-20 \%$ of the mean VO2peak.

In contrast, the LDCW is a relatively new functional performance measure with limited comparative studies. The LDCW and cardiorespiratory testing was performed in 102 healthy adults aged 60 to 91 years and showed a strong correlation between 400-meter time and VO2peak (-0.79) [24]. In a final model that also included stride length, ending systolic blood pressure, and a nonlinear correction factor, the 400 -meter time explained $62.5 \%$ of the variance in VO2peak. However, findings were not compared to the 6-MW. We found the correlation of VO2peak with the 400-meter time or the 6-MW distance to be similar in magnitude, though appropriately inversely related as shorter time and longer distance both represent better ambulatory function. Further, the workload, based on maximum heart rate, was not significantly different between the walk tests in our study. Our findings support the use of either the 6-MW or the 400-meter LDCW as surrogate research outcomes for VO2peak in HIV-infected patients. However, we agree with current clinical practice; medical decisions regarding individual therapy, such as surgical risk, should be based on direct measure of cardiorespiratory fitness [31].

The generalizability of the study is limited by the narrow demographic characteristics of the HIV-infected veterans at the Baltimore VA Medical Center, who are primarily African American men. Further, as part of CVD VACS, this study also excluded patients with prevalent CVD (CAD, PVD, and stroke) at baseline with the intent of following them longitudinally. Therefore it is not surprising 
Citation: Beans J, Stevenson T, Katzel LI, Sorkin JD, Warner AL, et al. (2013) Ambulatory Function in Men with and without HIV Infection: Association with Cardiorespiratory Fitness. J AIDS Clinic Res S9: 003. doi:10.4172/2155-6113.S9-003

that none of the participants had a 6 -MW distance $<350$ meters, a prognostic threshold in patients with heart failure and lung disease. Only 2 participants required more than 5.5 minutes to complete the 400-meter course of the LDCW. However, the use of younger and healthier patients would only bias the associations to the null. While the prevalence of comorbid conditions that could affect self-paced walking and VO2peak was similar between HIV-infected and uninfected men, disease duration and severity was not taken into consideration. The sample size limited our ability to test the association of comorbidity with function. Exercise physiologic research in a more diverse HIV patient population is warranted to investigate the mechanisms underlying loss of cardiorespiratory fitness and the impact on ambulatory function and mobility disability.

In conclusion, ambulatory function was not significantly associated with HIV infection in men with similar clinical characteristics, including absence of CVD history. The results from two different endurance walk tests provide the foundation for further research in a larger and more diverse patient population of HIV-infected adults to investigate agerelated factors that predict mortality and mobility disability. Walking tests may provide a valuable outcome for interventional trials, especially in specific groups of HIV-infected patients, such as those with heart and lung disease.

\section{Acknowledgements}

This work was supported by the National Institutes of Health (K23AG024896, R01HL095136, U01AA13566, P60AG12583, P30AG028747, R01HL090342) and the Baltimore VA Geriatric Research, Clinical and Education Center. Author roles included study concept and design (LK,MF,KO), statistical analysis (JS, KO), data interpretation, manuscript preparation, editing, and writing (all authors). We appreciate the research coordinators for their dedication, the participants for their time and Xiao-Song Zhong for assistance with compiling data.

\section{References}

1. Cesari M, Kritchevsky SB, Newman AB, Simonsick EM, Harris TB, et al. (2009) Added value of physical performance measures in predicting adverse healthrelated events: results from the Health, Aging And Body Composition Study. J Am Geriatr Soc 57: 251-259.

2. Studenski S, Perera S, Patel K, Rosano C, Faulkner K, et al. (2011) Gait speed and survival in older adults. JAMA 305: 50-58.

3. Newman AB, Simonsick EM, Naydeck BL, Boudreau RM, Kritchevsky SB, et al. (2006) Association of long-distance corridor walk performance with mortality, cardiovascular disease, mobility limitation, and disability. JAMA 295: 20182026.

4. Enright PL, McBurnie MA, Bittner V, Tracy RP, McNamara R, et al. (2003) The 6 -min walk test: a quick measure of functional status in elderly adults. Chest 123: 387-398.

5. Cahalin LP, Mathier MA, Semigran MJ, Dec GW, DiSalvo TG (1996) The sixminute walk test predicts peak oxygen uptake and survival in patients with advanced heart failure. Chest 110: 325-332.

6. Harada ND, Chiu V, Stewart AL (1999) Mobility-related function in older adults: assessment with a 6-minute walk test. Arch Phys Med Rehabil 80: 837-841.

7. Effros RB, Fletcher CV, Gebo K, Halter JB, Hazzard WR, et al. (2008) Aging and infectious diseases: workshop on HIV infection and aging: what is known and future research directions. Clin Infect Dis 47: 542-553.

8. Cote CG, Pinto-Plata V, Kasprzyk K, Dordelly LJ, Celli BR (2007) The 6-min walk distance, peak oxygen uptake, and mortality in COPD. Chest 132: 17781785.

9. Manini TM, Visser M, Won-Park S, Patel KV, Strotmeyer ES, et al. (2007) Knee extension strength cutpoints for maintaining mobility. J Am Geriatr Soc 55: 451-457.

10. Richert L, Dehail P, Mercié P, Dauchy FA, Bruyand M, et al. (2011) High frequency of poor locomotor performance in HIV-infected patients. AIDS 25: 797-805

11. Esposito JG, Thomas SG, Kingdon L, Ezzat S (2005) Anabolic growth hormone action improves submaximal measures of physical performance in patients with HIV-associated wasting. Am J Physiol Endocrinol Metab 289: E494-503.

12. Enright PL, Sherrill DL (1998) Reference equations for the six-minute walk in healthy adults. Am J Respir Crit Care Med 158: 1384-1387.

13. Oursler KK, Sorkin JD, Smith BA, Katzel LI (2006) Reduced aerobic capacity and physical functioning in older HIV-infected men. AIDS Res Hum Retroviruses 22: $1113-1121$.

14. Erlandson KM, Allshouse AA, Jankowski CM, Duong S, Mawhinney S, et al. (2012) Comparison of functional status instruments in HIV-infected adults on effective antiretroviral therapy. HIV Clin Trials 13: 324-334.

15. Thompson WR, American College of Sports Medicine, Gordon NF, Pescatello LS (2009) ACSM's Guidelines for Exercise Testing and Prescription. Lippincott Williams and Wilkins, Philadelphia.

16. Oursler KK, Katzel LI, Smith BA, Scott WB, Russ DW, et al. (2009) Prediction of cardiorespiratory fitness in older men infected with the human immunodeficiency virus: clinical factors and value of the six-minute walk distance. J Am Geriatr Soc 57: 2055-2061.

17. Duong M, Dumas JP, Buisson M, Martha B, Piroth L, et al. (2007) Limitation of exercise capacity in nucleoside-treated HIV-infected patients with hyperlactataemia. HIV Med 8: 105-111.

18. Pothoff G, Wassermann K, Ostmann H (1994) Impairment of exercise capacity in various groups of HIV-infected patients. Respiration 61: 80-85.

19. Terzian AS, Holman S, Nathwani N, Robison E, Weber K, et al. (2009) Factors associated with preclinical disability and frailty among HIV-infected and HIV-uninfected women in the era of cART. J Womens Health 18:1965-1974 (Larchmt) 18: 1965-1974.

20. Fried LP, Tangen CM, Walston J, Newman AB, Hirsch C, et al. (2001) Frailty in older adults: evidence for a phenotype. J Gerontol A Biol Sci Med Sci 56: M146-156

21. Cesari M, Onder G, Zamboni V, Manini T, Shorr RI, et al. (2008) Physical function and self-rated health status as predictors of mortality: results from longitudinal analysis in the iISIRENTE study. BMC Geriatr 8: 34.

22. Justice AC, Dombrowski E, Conigliaro J, Fultz SL, Gibson D, et al. (2006) Veterans Aging Cohort Study (VACS): Overview and description. Med Care 44: S13-24.

23. ATS Committee on Proficiency Standards for Clinical Pulmonary Function Laboratories (2002) ATS statement: guidelines for the six-minute walk test. Am J Respir Crit Care Med 166: 111-117.

24. Simonsick EM, Fan E, Fleg JL (2006) Estimating cardiorespiratory fitness in well-functioning older adults: treadmill validation of the long distance corridor walk. J Am Geriatr Soc 54: 127-132.

25. Grinspoon S, Corcoran C, Rosenthal D, Stanley T, Parlman K, et al. (1999) Quantitative assessment of cross-sectional muscle area, functional status, and muscle strength in men with the acquired immunodeficiency syndrome wasting syndrome. J Clin Endocrinol Metab 84: 201-206.

26. Oursler KK, Goulet JL, Leaf DA, Akingicil A, Katzel LI, et al. (2006) Association of comorbidity with physical disability in older HIV-infected adults. AIDS Patient Care STDS 20: 782-791.

27. Oursler KK, Goulet JL, Crystal S, Justice AC, Crothers K, et al. (2011) Association of age and comorbidity with physical function in HIV-infected and uninfected patients: results from the Veterans Aging Cohort Study. AIDS Patient Care STDS 25: 13-20.

28. Kwon S, Perera S, Pahor M, Katula JA, King AC, et al. (2009) What is a meaningful change in physical performance? Findings from a clinical trial in older adults (the LIFE-P study). J Nutr Health Aging 13: 538-544.

29. Erlandson KM, Allshouse AA, Jankowski CM, Duong S, MaWhinney S, et al. (2012) Risk factors for falls in HIV-infected persons. J Acquir Immune Defic Syndr 61: 484-489.

30. Ross RM, Murthy JN, Wollak ID, Jackson AS (2010) The six minute walk test accurately estimates mean peak oxygen uptake. BMC Pulm Med 10: 31

31. Cahalin L, Pappagianopoulos P, Prevost S, Wain J, Ginns L (1995) The relationship of the 6-min walk test to maximal oxygen consumption in transplant candidates with end-stage lung disease. Chest 108: 452-459. 